\title{
Bem-estar animal: conceitos e formas práticas de avaliação dos sistemas de produção de suínos
}

\section{Animal welfare: concepts and practical procedures to evaluate the swine productions systems}

\author{
Xavier Manteca ${ }^{1}$; Caio Abércio da Silva²; Ana Maria Bridi²; \\ Cleandro Pazinato Dias ${ }^{3^{*}}$
}

\begin{abstract}
Resumo
As definições de bem-estar animal têm sido amplamente debatidas junto à comunidade científica internacional durante as últimas décadas. A linha conceitual mais aceita trata do bem-estar animal dentro de um enfoque multidimensional, abrangendo emoções, funcionamento biológico e comportamento natural. No entanto, compreendido os conceitos, outro desafio que se apresenta é como mensurar adequadamente o bem-estar animal nas condições de campo. Neste sentido, o projeto Welfare Quality ${ }^{\circledR}$ fornece uma compreensão muito útil dos componentes de bem-estar e de como podemos avaliá-lo em granjas comerciais e abatedouros de suínos. O objetivo deste artigo é discorrer sobre os principais conceitos científicos de bem-estar animal e sobre as formas práticas de avaliação segundo os protocolos do projeto Welfare Quality ${ }^{\circledR}$ em suínos, identificando seus aspectos positivos e críticos.
\end{abstract}

Palavras-chave: Mensurações, suinocultura, Welfare Quality ${ }^{\circledR}$

\begin{abstract}
The concepts of animal welfare have received a wide discussion by international scientific community during the last decades. The most accepted definition consider the animal welfare like a multidimensional theme, where are involved the emotion, the biological activity and the natural behavior. However, considering the clearness of this concepts, another challenge is presented, where is questioned how we can evaluate the animal welfare in commercial systems. Thus, the Welfare Quality ${ }^{\circledR}$ project gave the understanding about the welfare subjects and how we can to evaluate its in commercial farms and slaughterhouses. Then the main of this article is present the principal concepts and practical means to evaluate the animal welfare in swine, according the Welfare Quality ${ }^{\circledR}$ protocols, with the identifications of the positive and critical aspects involved.
\end{abstract}

Key words: Measurements, swine production, Welfare Quality ${ }^{\circledR}$

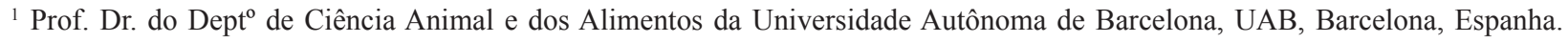
E-mail: xavier.manteca@uab.cat

${ }^{2}$ Profs. Drs. do Detpt ${ }^{\circ}$ de Zootecnia, Universidade Estadual de Londrina, UEL, Londrina, PR. E-mail: casilva@uel.br; ambridi@ uel.br

${ }^{3}$ Discente de Doutorado, Programa de Pós-Graduação em Ciência Animal, UEL, Londrina, PR. E-mail: cleandropazinato@uol. com.br

* Autor para correspondência 


\section{Conceitos de bem-estar animal}

$\mathrm{Na}$ discussão do bem-estar animal a definição do próprio conceito constitui o primeiro desafio do tema, dada à complexidade do assunto e a grande divergência observada entre os cientistas que atuam na área.

Segundo Hughes (1982), o bem-estar corresponde a um estado onde o animal está em harmonia com a natureza ou com o seu ambiente. A este conceito Hurnik (1992) adicionou a ideia de que o bem-estar significa uma alta qualidade de vida do animal, defendendo que um ótimo funcionamento biológico do organismo ocorre somente quando a sua vida está identificada ou alinhada com o ambiente, reiterando que este cenário denomina-se estado de harmonia. Também considera que somente nestas condições, com o melhor funcionamento biológico, é que o animal alcança sua máxima qualidade de vida. No entanto, Broom (2011) acrescenta que esta definição, embora seja amplamente utilizada e represente uma das mais importantes formas de conceituar o tema, tem uma aplicação científica limitada, pois não permite que com facilidade possa ser mensurado este estado de harmonia.

Anteriormente, Broom (1986) apresentou uma definição que foi muito bem aceita e difundida, defendendo que o bem-estar de um indivíduo é o seu estado em relação às suas tentativas de lidar (em uma tradução livre da palavra inglesa cope) com o ambiente em que se encontra. $\mathrm{O}$ autor destacou que quando os desafios são vencidos com pouco esforço e gasto de recursos, o bem-estar do indivíduo é satisfatório. Por outro lado, quando o indivíduo falha no enfrentamento destes desafios, o bem-estar pode ser considerado pobre.

Considera-se que os desafios potencialmente prejudiciais podem ser provenientes de condições alheias ao indivíduo, como os agentes patogênicos ou as estruturas que venham a causar danos nos tecidos, como um piso muito abrasivo para o casco, por exemplo; ou a partir do próprio indivíduo, em decorrência do ambiente, como o estado de tédio, a ansiedade ou a frustração (BROOM, 2001b).

Neste contexto, Broom e Johnson (1993) identificaram três aspectos relacionados com a questão: i) o bem-estar é uma característica do animal e não algo que o homem pode fornecer; ii) o bem-estar pode variar de muito bom a muito ruim; iii) o bem-estar pode ser medido cientificamente.

Para Broom (2011) o bem-estar animal é um conceito científico que descreve uma qualidade de vida potencialmente mensurável de um ser vivo em um determinado momento, no entanto, ressalta que a abordagem científica do tema deve estar amplamente separada da ética. O bem-estar deve ser medido de forma objetiva, com uma avaliação completamente isenta de considerações éticas, devendo prover as informações necessárias para que as decisões éticas possam ser tomadas em situações específicas (BROOM; MOLETO, 2004).

Ao relacionar o termo saúde com o bemestar deve-se compreender que o mesmo se refere a um estado de harmonia, de equilíbrio dos sistemas corporais que participam do combate aos patógenos, da recuperação dos danos teciduais e/ ou dos transtornos fisiológicos. Portanto, a saúde pode ser definida como um estado positivo do animal em relação às tentativas de enfrentar uma patologia. Assim, a saúde pode ser inserida no bemestar, sendo ambos qualificados em uma escala que varia entre o muito bom e o muito ruim (BROOM; MOLETO, 2004; BROOM, 2011). A saúde, todavia, não é sinônimo de bem-estar, mas sim um de seus componentes, sendo que o estado caracterizado como saudável é uma condição essencial para um bom bem-estar animal.

Na visão de Duncan e Fraser (1997), a maioria das definições de bem-estar animal está agrupada em uma das três escolas de pensamento, aquelas baseadas nas emoções dos animais, aquelas com base no funcionamento biológico do organismo animal, e aquelas relacionadas com o comportamento "natural" dos animais.

No grupo das definições baseadas nas emoções 
o bem-estar é considerado reduzido nos estados das emoções negativas, como a dor, medo, frustração, fome e sede, e aumentado nos estados de emoções positivas, como conforto, contentamento e o prazer obtido em certos tipos de interação social (DUNCAN; FRASER, 1997), estados estes comumente denominados de sofrimento e prazer, respectivamente (DUNCAN, 2005).

Uma das peculiaridades desta escola é acreditar na capacidade dos animais experimentarem sofrimentos (DUNCAN, 1996) e em considerar os sentimentos subjetivos dos mesmos, particularmente os sentimentos desagradáveis (DAWKINS, 1988).

Para aqueles que defendem esta linha de pensamento o desafio desta ciência está em estudar e entender o sentimento e as emoções dos animais, que são experiências subjetivas e que não podem ser observadas diretamente. Neste sentido, foram desenvolvidos testes de preferência que correspondem a "questionamentos" dirigidos aos animais que buscam identificar o que eles sentem, assumindo que os mesmos escolherão o que melhor atenderá seu bem-estar. Associados a estas avaliações devem ser empregados testes "motivacionais", que são orientados para medir o quão importante é a escolha estabelecida pelo animal (DUNCAN, 2005). Todavia, pelas dificuldades de interpretação destes testes, que envolvem o estudo das emoções, muitos pesquisadores preferem usar medidas mais tradicionais, baseadas na funcionalidade do corpo (DUNCAN; FRASER, 1997).

As definições baseadas no funcionamento biológico estabelecem que o bem-estar será reduzido pelas doenças, injúrias e pela má nutrição, e, por outro lado, o bom bem-estar será indicado pelos altos níveis de crescimento e reprodução, funcionamento normal dos processos fisiológicos e comportamentais, e altas taxas de longevidade e aptidão física (DUNCAN; FRASER, 1997). Muitos pesquisadores que adotam estas definições são influenciados pelo conceito de que se o estresse aumenta o bem-estar será reduzido.
Neste sentido, o estresse pode ser definido como um estímulo ambiental com potencial para sobrecarregar ou desafiar os sistemas de controle ou de homeostasia do animal, reduzindo sua adaptação (BROOM, 2003; citado por BROOM, 2008). A palavra estresse deveria ser utilizada para descrever aquela porção do bem-estar pobre, que se refere somente às situações nas quais houve a falência das tentativas de enfrentar as dificuldades (BROOM; MOLENTO, 2004).

O termo homeostase é empregado para determinar a manutenção do equilíbrio ou o estado de constância do meio interno que, em essência, é decorrente das funções desempenhadas por todos os órgãos e tecidos do corpo (GUYTON; HALL, 1997). Assim, o bem-estar é comprometido quando o animal não consegue manter a homeostase ou quando a mantém sob elevado dispêndio de energia. A manutenção da homeostase se dá através dos diferentes sistemas de controle do animal, que quando estimulados têm a necessidade de recorrer a recursos para atendê-los. O termo necessidade, neste contexto, é entendido como um requerimento que faz parte da biologia básica do animal para obter um recurso particular ou para responder a um ambiente em especial ou a um estímulo corporal (BROOM, 2001a). Assim, o bem-estar é prejudicado quando o animal não consegue satisfazer-se para uma determinada necessidade.

A escola baseada no funcionamento biológico acredita que o bem-estar está intimamente ligado à ausência de uma resposta fisiológica ao estresse, ou pelo menos à ausência de uma ampla resposta, pois quando o animal é confrontado com uma alteração no ambiente, a adaptação envolve uma série de respostas fisiológicas para manter a homeostase. Estas respostas podem ser agudas ou crônicas dependendo da duração da ativação do eixo hipotálamo-hipófise-adrenal. Quando estas se cronificam desviam recursos de outras atividades, como reprodução e crescimento, podendo também levar à imunossupressão (BARNETT; HEMSWORTH, 1990). 
Uma revisão sobre as respostas ao estresse, realizada por Bergeron, Meunier-Salaun e Robert (2008), identificou que fêmeas suínas gestantes mantidas amarradas apresentaram um aumento do cortisol basal e um aumento do hormônio adrenocorticotrófico e do fator liberador de cortisol, quando comparadas com fêmeas alojadas individualmente (em gaiolas ou em baias grandes o suficiente para desenvolverem qualquer movimento voluntário) ou alojadas coletivamente em baias. Estes resultados foram usados como uma base científica em muitos países para o banimento do uso das correntes para contenção de matrizes.

Efetivamente há uma dificuldade na definição sobre o limite que separa uma perda de bemestar animal de um ajuste normal das taxas destes hormônios indicadores de estresse (HEMSWORTH, 1990; citado por DUNCAN; FRASER, 1997). O cortisol é relativamente fácil de ser medido, mas os valores sofrem grandes variações ao longo do dia e o próprio ato da coleta das amostras também pode interferir nos resultados (McGLONE et al., 2004).

Portanto, avaliar o bem-estar animal baseado em dados, considerando a atividade do eixo hipotálamohipófise-adrenal, deve ser visto com cautela, devido à inconsistência entre os resultados de diferentes estudos. Amostras adicionais de sangue também não conferem uma acurada descrição de episódios naturais de secreção de corticosteroides (RUSHEN, 1991).

Dentro deste grupo de definições foram propostas que as necessidades biológicas dos animais poderiam ser hierarquizadas em três categorias, em ordem de peso e importância decrescente sobre a qualidade de vida, sendo primeiramente listadas aquelas vinculadas à manutenção da vida, seguidas pelas de manutenção da saúde, e finalmente pelas necessidades de manutenção do conforto (HURNIK, 1988).

O grupo de definições baseado no comportamento "natural" considera que para promover o bem-estar os animais devem estar em um ambiente que permita que expressem ao máximo o comportamento natural da espécie. Cientistas que aplicam este conceito frequentemente estudam o comportamento dos animais na natureza e o comparam com o comportamento dos animais em cativeiro, supondo que as diferenças observadas são decorrentes do ambiente (DUNCAN; FRASER, 1997).

A visão de que os animais deveriam manifestar todo o seu repertório de comportamentos foi compartilhada nas primeiras pesquisas de bemestar animal. Posteriormente foram entendidas que parte deste repertório incluía certas atividades que decorriam ou envolviam a adaptação dos animais às circunstâncias adversas encontradas. Os animais na natureza sofrem de situações ameaçadoras com severos e frequentes problemas com risco de morte, e seu comportamento natural reflete seu esforço para sobreviver. Assim seria incorreto considerar que o comportamento selvagem (natural) é sempre indicativo de bem-estar. Muitas vezes este comportamento pode representar a luta pela sua sobrevivência (POOLE, 1996).

No entanto, algumas necessidades de comportamento são imprescindíveis para a manutenção do bem-estar dos suínos, pois quando não são atendidas alteram a produção e causam padecimento mental. As necessidades de comportamento em que o suíno é altamente motivado envolvem: i) necessidade de exploração e busca de alimento; ii) necessidade de locomoção; iii) necessidade de construção do ninho antes do parto; iv) necessidade de contato social (BERGERON; MEUNIER-SALAUN; ROBERT, 2008). O não reconhecimento da importância das necessidades comportamentais pode ser contrário à maximização da produtividade, pois tem sido aceito que desempenhar os comportamentos típicos da espécie contribui para a aptidão biológica do animal. No entanto, para demonstrar que uma necessidade é realmente verdadeira, deve ser mostrado que a falta do atendimento da mesma resulta em comprometimento do bem-estar, com consequências negativas quando essas ações 
não podem ser realizadas de forma satisfatória (BAXTER; LAWRENCE; EDWARDS, 2011).

Considerando um enfoque multidimensional, o bem-estar animal pode ser caracterizado de diferentes maneiras, mas existe um consenso que em qualquer definição deva ser considerado três aspectos: o estágio emocional do animal, o funcionamento biológico e a habilidade do animal mostrar o padrão normal de comportamento (MANTECA; VELARDE; JONES, 2009). Apesar destes três conceitos de bem-estar serem a princípio diferentes, não são contraditórios e frequentemente são complementares. A compreensão desta interrelação é verificada nos estudos que demonstram que diante da impossibilidade do desenvolvimento de uma conduta comportamental importante o animal experimenta uma resposta ao estresse, que pode ser identificada finalmente através dos parâmetros fisiológicos (MANTECA; GASA, 2008). As cinco liberdades desenvolvidas pelo Farm Animal Welfare Council (FAWC, 2009) combinam as três abordagens descritas e apresentam um enfoque prático e integrador, propondo que o bem-estar animal estaria adequado quando são cumpridas as seguintes condições: nutrição adequada; conforto térmico e físico; ausência de enfermidades e lesões; possibilidade de expressar as condutas próprias da espécie, sobretudo aquelas em que o animal demonstra forte motivação; e ausência de dor ou estresse intenso ou duradouro (MANTECA, 2011).

A Organização Mundial de Saúde Animal (OIE, 2009) trata que bem-estar animal corresponde à forma como o animal lida com o seu entorno. Um animal está em boas condições de bem-estar se estiver saudável, confortável, bem alimentado, seguro, apto para expressar suas formas inatas de comportamento e livre de dor, medo ou angústia. As boas condições de bem-estar exigem a prevenção de enfermidades e a administração de tratamentos veterinários apropriados mais abrigo, alimentação, manejo e abate humanitário.

Outro enfoque integrador foi proposto pelo projeto Welfare Quality ${ }^{\circledR}$, definindo o bem-estar com base em quatro princípios, considerando como estes são experimentados pelos animais, sendo: boa alimentação, bom alojamento, boa saúde e expressão de um comportamento adequado (BOTREAU et al., 2007b). Estes princípios estão baseados nos seguintes questionamentos: i) os animais estão bem alimentados e abastecidos com água? ii) os animais estão devidamente alojados? iii) os animais estão saudáveis? iv) o comportamento dos animais reflete estados emocionais adequados? (BLOKHUIS, 2008).

\section{Como Medir Bem-Estar Animal Segundo o Projeto Welfare Quality ${ }^{\circledR}$}

O Projeto Welfare Quality ${ }^{\circledR}$ obteve financiamento da Comissão Europeia e envolveu especialistas de 44 Institutos e Universidades oriundos de 13 países europeus e de quatro da América Latina. Este projeto integrado de pesquisa foi iniciado em maio de 2004 e foi finalizado em dezembro de 2009 (VELARDE; DALMAU, 2012). A preocupação dos consumidores e a aparente demanda por informações sobre bem-estar animal foi o ponto de partida para a União Europeia financiar o projeto (BLOKHUIS, 2007).

Os objetivos primários do projeto Welfare Quality ${ }^{\circledR}$ foram desenvolver estratégias práticas para melhorar o bem-estar animal; elaborar um protocolo para avaliar o bem-estar em granjas e abatedouros; definir um protocolo para informar nos produtos de origem animal a condição de bem-estar; e integrar e inter-relacionar especialistas multidisciplinares do bem-estar animal na Europa (BLOKHUIS, 2008). Contudo, o objetivo principal foi desenvolver uma avaliação global do bem-estar animal a fim de dar suporte aos consumidores quanto às informações sobre os produtos que são disponibilizados para consumo (BLOKHUIS et al., 2003).

O projeto Welfare Quality ${ }^{\circledR}$ reconheceu que os consumidores identificaram que a qualidade de um alimento não era determinada somente 
pela condição de ser natural ou segura, mas pela condição de bem-estar a que eram submetidos os animais que produziam estes, integrando assim a visão do bem-estar animal ao conceito da qualidade do alimento (BLOKHUIS, 2008). Portanto, esta nova percepção quebrou um paradigma que perdurou por anos, de que a produção agropecuária estava focada no preço da oferta e na concorrência (BLOKHUIS et al., 2003).

Paralelamente, considerando a multidisciplinaridade do tema (FRASER, 1995), o aprofundamento dos conhecimentos da ciência do bem-estar, a diversidade de recursos metodológicos para sua identificação/qualificação, e que a característica multidimensional do tema requer parâmetros externos para sua avaliação (BLOKHUIS, 2008), o projeto Welfare Quality ${ }^{\circledR}$ ganhou todo o apoio e motivação para ser desenvolvido. Estas foram, portanto, as razões da necessidade de integração de especialistas para desenvolver, aperfeiçoar, padronizar e calibrar os sistemas de avaliação de bem-estar, e também para identificar e validar as medidas corretivas (BLOKHUIS, 2008).

Anterior ao projeto Welfare Quality ${ }^{\circledR}$ alguns procedimentos metodológicos diretos foram propostos para prover uma avaliação global do bem-estar, como a avaliação sérica dos níveis de corticosteroides e de proteínas de fase aguda e a medição da longevidade do animal. Contudo, muitos questionamentos eram feitos, considerando que estas condutas não eram suficientes para atender e explicar as dimensões do bem-estar. Segundo Botreau et al. (2007a), um animal poderia estar doente sem comprometimento da atividade do eixo hipotálamo-hipófise-adrenal, e vice-versa, ou seja, várias medidas são necessárias para obter uma visão abrangente do bem-estar animal.

Preservados naturalmente as qualidades destas medidas precursoras, outros modelos de avaliação de bem-estar, baseados na composição de várias medidas simultaneamente obtidas numa única avaliação, foram sugeridos. Estes modelos orientam que os dados obtidos em uma unidade possam ser analisados por um especialista, que extrai uma conclusão geral; que os dados sejam passíveis de serem comparados com os requerimentos mínimos estabelecidos para cada medida; que possam ser convertidos em um ranking; e que possam ser então somados e finalmente convertidos em valores ou pontuações (escores) resultantes de uma soma ponderada ou usando regras ad hoc. No entanto, entre as críticas a este processo estão a sua baixa sensibilidade e o fato de não serem aplicáveis rotineiramente, por não refletirem a natureza multidimensional do bem-estar e a relativa importância das várias medidas de bem-estar (BOTREAU et al., 2007a).

Os protocolos de avaliação do projeto Welfare Quality ${ }^{\circledR}$ foram elaborados por um amplo diálogo entre cientistas da área animal, sociólogos e sociedade, contemplando numerosas interações resultantes de reuniões, conferências, oficinas, sites interativos, boletins, entrevistas, grupos de discussão, júris etc. Os quatro momentos chave deste diálogo foram a elaboração da lista inicial com 12 critérios de bem-estar; os grupos de discussão; o desenvolvimento do sistema de escores; e os júris de cidadãos (MIELE et al., 2011). Os modelos foram desenvolvidos para as espécies bovina, suína e aves de postura e frangos de corte, para aplicação em sistemas de produção europeus (BLOKHUIS et al., 2003).

O projeto Welfare Quality ${ }^{\circledR}$ combinou análises de percepção e atitudes do consumidor com o conhecimento existente da ciência do bem-estar animal, identificando quatro princípios e 12 critérios que devem ser adequadamente cobertos nos sistemas de avaliação (KEELING; VEISSIER, 2005) (Tabela 1). 
Tabela 1. Princípios e critérios do sistema de avaliação de bem-estar animal do projeto Welfare Quality ${ }^{\circledR}$.

\begin{tabular}{lll}
\hline Princípios & \multicolumn{2}{l}{ Critérios } \\
\hline 1. Boa alimentação & 1. & Ausência de fome prolongada \\
2. Bom alojamento & 2. & Ausência de sede prolongada \\
& 3. & Conforto em relação ao descanso \\
& 4. & Conforto térmico \\
3. Boa saúde & 5. & Facilidade de movimento \\
& 6. & Ausência de lesões \\
& 7. & Ausência de enfermidades \\
4. Comportamento adequado & 8. & Ausência de dor causada por práticas de manejo \\
& 9. & Expressão de comportamento social adequado \\
& 10. & Expressão adequada de outras condutas \\
& 11. & Interação humano animal positiva \\
& 12. & Estado emocional positivo \\
\hline
\end{tabular}

Fonte: Adaptado Keeling e Veissier (2005).

Os princípios básicos dos protocolos do projeto Welfare Quality ${ }^{\circledR}$ seguiram uma versão modificada do princípio das cinco liberdades, agrupando os mesmos em quatro princípios, que são: boa alimentação, bom alojamento, boa saúde e adequado comportamento (BOTREAU et al., 2007b).

Algumas características específicas relacionadas com o conceito de bem-estar que foram assumidas no projeto são representadas pelos seguintes pontos i) o bem-estar é um conceito multidimensional; ii) o bem-estar animal é considerado em um nível individual, embora seja geralmente uma avaliação global, definido em nível de granja; iii) uma dimensão do bem-estar não pode ser totalmente compensada por outra; iv) o bem-estar animal é interpretado por humanos, baseado em evidências científicas, opinião de especialistas e visão de stakeholders (BOTREAU et al., 2007b).

O projeto Welfare Quality ${ }^{\circledR}$ desenvolveu protocolos padronizados de avaliação que utilizam várias medidas, incluídas nos quatro princípios e nos 12 critérios (Tabela 1). Este sistema objetivou constituir, portanto, em uma ferramenta útil, direta, prática e ampla para informar os diversos agentes da cadeia produtiva (desde o fornecedor até o cliente) do status de bem-estar animal.

$\mathrm{Na}$ espécie suína o projeto Welfare Quality ${ }^{\circledR}$ desenvolveu três protocolos distintos de avaliação, dois para serem aplicados nas granjas, sendo um em porcas e leitões e outro em suínos em fase de crescimento, e um protocolo para ser aplicado em suínos de engorda que são dirigidos ao abatedouro (WELFARE QUALITY ${ }^{\circledR}, 2009$ ).

Para cada critério medidas potenciais foram identificadas e consideradas para inclusão nas avaliações de bem-estar, levando-se em conta sua validade (validity), repetibilidade (reliability) e viabilidade (feasibility) (VELARDE; DALMAU, 2012). O projeto, portanto, organizou as medidas existentes na literatura, avaliou novas metodologias e buscou uma combinação destas, cumprindo as condições bases estabelecidas: i) que as medidas sejam válidas, ou seja, que realmente sejam úteis para medir algum aspecto do bem-estar; ii) que as medidas sejam repetíveis, ou seja, que diferentes observadores avaliando um mesmo animal obtenham um resultado parecido, e um mesmo observador avaliando duas vezes o mesmo animal também obtenha resultados semelhantes; iii) que as medidas sejam práticas, ou seja, que não necessitem de muitos equipamentos nem demandem demasiado tempo para determinarem um resultado, que se ajustem às condições de alojamento dos animais, que eliminem medidas que tenham que ser submetidas 
à análise laboratorial ou que requeiram muitos equipamentos; iv) priorizem medidas baseadas no animal, ou seja, que não utilizem medidas de bemestar a partir de indicadores indiretos (baseados em instalações e manejo), priorizando os parâmetros obtidos diretamente dos animais (ex: lesões); v) que utilize medidas universais, ou seja, aplicáveis em todos os sistemas de produção (intensivos e extensivos); vi) que a combinação final obtenha uma pontuação global do bem-estar de forma multidimensional e equilibrada (TEMPLE et al., 2009).

As medidas relacionadas com o ambiente, que têm relação com o bem-estar animal, têm foco na identificação das causas do pobre bem-estar e na proposição de medidas corretivas (feedback para o produtor). Um ponto de partida fundamental no projeto Welfare Quality ${ }^{\circledR}$ é que parâmetros baseados nos animais são mais válidos para avaliar o bemestar, considerando, portanto, que o bem-estar animal é de propriedade do animal e que estas medidas podem incluir os efeitos das variações da forma como o sistema de produção é gerenciado (papel do produtor), bem como das interações específicas animal/sistema (BRACKE et al., 2009).

As observações baseadas no animal fornecem informações mais diretas sobre como os animais estão se adaptando ao ambiente, dando uma indicação da performance do sistema de criação em um determinado momento do ciclo produtivo. Contudo, as observações baseadas nos recursos não devem ser negligenciadas, pois são essenciais para prover recomendações para prever problemas de bem-estar e para a avaliação de riscos de deficientes bem-estar (TEMPLE et al., 2012b).

Em relação aos tipos de medidas, o projeto Welfare Quality ${ }^{\circledR}$ definiu três classes: i) aquelas baseadas no animal, obtidas diretamento destes; ii) medidas baseadas no manejo, obtidas com o proprietário/gerente da unidade e nos processos de gestão que são empregados; iii) medidas baseadas em recursos, que são aquelas obtidas do ambiente em que os animais são mantidos (WELFARE QUALITY $\left.^{\circledR}, 2009\right)$.

As medidas baseadas no animal podem ser usadas efetivamente como ferramentas para a avaliação do bem-estar em granjas com relação às leis, programas de boas práticas, esquemas de garantia de qualidade e também para avaliar o manejo (EFSA, 2012a). Correspondem a apropriados indicadores de bemestar, e sua combinação baseada na seleção de medidas seguras pode ser usada para medir um estado de bem-estar de uma população alvo de uma forma válida e robusta (EFSA, 2012b).

O sistema de avaliação do projeto Welfare Quality $^{\circledR}$ valoriza principalmente as medidas baseadas no animal (também chamadas de medidas outcome ou de performance), em detrimento das medidas basedas em recursos ou manejo. Esta conduta busca estimar o estatus real do bem-estar dos animais e envolve aspectos de caracter fisiológico, sanitário e comportamental, apresentando vantagens em relação às demais medidas, pois avaliam o bem-estar diretamente do animal e são aplicáveis em qualquer granja ou abatedouro (VELARDE; DALMAU, 2012).

Considera-se que os indicadores baseados no animal abrangem todas as circunstâncias relacionadas com a espécie vinculadas com as características da criação, as condições de espaço e alojamento, as possibilidades de comportamento natural, as condutas veterinária, o desmame, as intervenções cirúrgicas etc., incluindo também o transporte até o abatedouro, a insensibilização e o sacrifício (UNIÓN EUROPEA, 2011).

As avaliações de bem-estar baseadas no animal refletem as práticas de manejo erradas, as negligências e os abusos com os animais e os equipamentos mal concebidos. Os protocolos baseados no ambiente devem ser evitados, pois podem limitar o desenvolvimento de métodos inovadores. Assim, poucas medidas com este escopo deveriam ser usadas, apenas aquelas necessárias para manter um nível mínimo aceitável de bem- 
estar (como a amperagem mínima nos sistemas de insensibilização elétrica). Avaliações que têm como referência os registros não deveriam ser aplicadas pelo risco de falsificação que guardam (GRANDIN, 2010). Assim, o melhor sistema de avaliação é aquele que utiliza a observação direta do animal.

As Tabelas 2, 3, 4, e 5 apresentam as diferentes medidas, tipos de medidas e os critérios utilizados nos três protocolos Welfare Quality ${ }^{\circledR}$ aplicados em granjas e abatedouro de suínos.

Tabela 2. Medidas, tipo da medida e critérios obtidos na granja aplicadas no protocolo de porcas e leitões do projeto Welfare Quality ${ }^{\circledR}$.

\begin{tabular}{|c|c|c|}
\hline Medidas & Tipo da medida & Critérios \\
\hline Escore da condição corporal & Baseada no animal & Ausência de fome prolongada \\
\hline Idade de desmame & Baseada no manejo & Ausência de fome prolongada \\
\hline Suprimento de água & Baseada em recurso & Ausência de sede prolongada \\
\hline $\begin{array}{l}\text { Bursites (injúrias por pressão), feridas nos ombros } \\
\text { (injúrias por pressão), fezes aderidas no corpo }\end{array}$ & Baseada no animal & Conforto em relação ao descanso \\
\hline Ofegação, amontoamento & Baseada no animal & Conforto térmico \\
\hline Densidade/animal, celas parideiras & Baseada em recurso & Facilidade de movimento \\
\hline Claudicação, feridas no corpo, lesões de vulva & Baseada no animal & Ausência de lesões \\
\hline Mortalidade & Baseada no manejo & Ausência de enfermidades \\
\hline $\begin{array}{l}\text { Tosse, espirro, batedeira (respiração laboriosa), } \\
\text { prolapso retal, escore diarreia, constipação, metrites, } \\
\text { mastites, prolapso uterino, condição da pele, rupturas } \\
\text { e hérnias, infecções locais, desordens neurológicas } \\
\text { (tremor), splay leg (membros abertos) }\end{array}$ & Baseada no animal & Ausência de enfermidades \\
\hline $\begin{array}{l}\text { Destrompe do focinho e corte/cauda, castração, corte/ } \\
\text { cauda e corte/dentes }\end{array}$ & Baseada no manejo & $\begin{array}{l}\text { Ausência de dor causada por } \\
\text { práticas de manejo (mutilações) }\end{array}$ \\
\hline Comportamento social (positivo/negativo) & Baseada no animal & $\begin{array}{l}\text { Expressão de comportamento } \\
\text { social adequado }\end{array}$ \\
\hline Estereotipias, comportamento exploratório & Baseada no animal & $\begin{array}{l}\text { Expressão adequada de outras } \\
\text { condutas }\end{array}$ \\
\hline Medo dos humanos & Baseada no animal & Interação humano animal positiva \\
\hline Avaliação qualitativa do comportamento (QBA) & Baseada no animal & Estado emocional positivo \\
\hline
\end{tabular}

Fonte: Adaptado Welfare Quality ${ }^{\circledR}$ (2009). 
Tabela 3. Medidas, tipo da medida e critérios obtidos na granja aplicadas no protocolo de suínos em crescimento ${ }^{1}$ do projeto Welfare Quality ${ }^{\mathbb{B}}$ :

\begin{tabular}{lll}
\hline Medidas & Tipo da medida & Critérios \\
\hline Escore da condição corporal & Baseada no animal & Ausência de fome prolongada \\
Suprimento de água & Baseada em recurso & Ausência de sede prolongada \\
Bursites (injúrias por pressão), fezes aderidas no corpo & Baseada no animal & Conforto em relação ao descanso \\
Tremor de frio, ofegação, amontoamento & Baseada no animal & Conforto térmico \\
Densidade/animal & Baseada em recurso & Facilidade de movimento \\
Claudicação, feridas no corpo, caudofagia & Baseada no animal & Ausência de lesões \\
Mortalidade & Baseada no manejo & Ausência de enfermidades \\
$\begin{array}{l}\text { Tosse, espirro, batedeira (respiração laboriosa), focinho } \\
\text { torcido, prolapso retal, escore diarreia, condição da pele, } \\
\text { rupturas e hérnias }\end{array}$ & Baseada no animal & Ausência de enfermidades \\
Castração, corte/cauda & Baseada no manejo & $\begin{array}{l}\text { Ausência de dor causada por práticas de } \\
\text { manejo (mutilações) }\end{array}$ \\
Comportamento social (positivo/negativo) & Baseada no animal & $\begin{array}{l}\text { Expressão de comportamento social } \\
\text { adequado }\end{array}$ \\
Comportamento exploratório & Baseada no animal & Expressão adequada de outras condutas \\
Medo dos humanos & Baseada no animal & Interação humano animal positiva \\
Avaliação qualitativa do comportamento (QBA) & Baseada no animal & Estado emocional positivo \\
\hline
\end{tabular}

1: Suínos em crescimento: suínos criados com o propósito de produzir carne ou reprodução, do desmame até estarem prontos para o abate.

Fonte: Adaptado Welfare Quality ${ }^{\circledR}$ (2009).

Algumas medidas relacionadas com a saúde são obtidas no abatedouro, no período pós-mortem, e refletem as condições da granja em que estes suínos estavam sendo criados. A análise das mesmas deve ser feita conjuntamente com a medidas realizadas na granja.

Tabela 4. Medidas, tipo da medida e critério obtidos no abatedouro aplicadas no protocolo de suínos em crescimento do projeto Welfare Quality ${ }^{\circledR}$

\begin{tabular}{lll}
\hline Medidas & Tipo da medida & Critério \\
\hline Pneumonia, pleurisia, pericardite, manchas brancas no fígado & Baseada no animal & Ausência de enfermidades \\
\hline
\end{tabular}

Fonte: Adaptado Welfare Quality ${ }^{\circledR}$ (2009). 
Tabela 5. Medidas, tipo da medida e critérios obtidos no abatedouro aplicadas no protocolo de suínos em terminação ${ }^{1}$ do projeto Welfare Quality ${ }^{\circledR}$ :

\begin{tabular}{lll}
\hline Medidas & Tipo da medida & Critérios \\
\hline $\begin{array}{l}\text { Provisão de ração } \\
\text { Suprimento de água } \\
\text { Qualidade do piso, cama dos caminhões }\end{array}$ & $\begin{array}{l}\text { Baseada em recurso } \\
\text { Baseada em recurso } \\
\text { Baseada no manejo e } \\
\text { recurso }\end{array}$ & $\begin{array}{l}\text { Ausência de fome prolongada } \\
\text { Ausência de sede prolongada } \\
\text { Conforto em relação ao descanso }\end{array}$ \\
$\begin{array}{l}\text { Tremor de frio, ofegação, amontoamento } \\
\text { Escorregamento/deslizamento, queda }\end{array}$ & $\begin{array}{l}\text { Baseada no animal no animal } \\
\text { Densidade animal na carga, densidade animal na } \\
\text { baia de espera }\end{array}$ & $\begin{array}{l}\text { Conforto térmico } \\
\text { Facilidade de movimento }\end{array}$ \\
$\begin{array}{l}\text { Animaimaicação, feridas no corpo no manejo } \\
\text { Efetividade da insensibilização }\end{array}$ & Facilidade de movimento \\
& Baseada no animal & Ausência de lesões \\
Vocalizações (alta frequência) & Baseada no animal & Ausência de enfermidades \\
Relutância em mover-se, retroceder & Baseada no animal & $\begin{array}{l}\text { Ausência de dor causada por } \\
\text { práticas de manejo }\end{array}$ \\
\hline
\end{tabular}

1: Suínos em terminação: são os mesmos suínos de crescimento agora no abatedouro prontos para o abate.

Fonte: Adaptado Welfare Quality ${ }^{\circledR}$ (2009).

Com a finalidade de aumentar a repetibilidade e confiabilidade da avaliação, as medidas devem apresentar facilidade de coleta, de obtenção, ao mesmo tempo em que seja minimizado o julgamento de seu valor pelo observador. A maioria das medidas é avaliada segundo uma escala de três pontos (variando 0 a 2). O escore zero (0) é atribuído às observações onde o bem-estar é considerado bom. Quando houver algum comprometimento do bem-estar, o 1 é aplicado; e o escore 2 é referente ao pobre ou ao inaceitável bem-estar. Em alguns casos usam-se escalas binárias ( $0 / 2$ ou sim/não) ou cardinais (por exemplo, cm ou $\mathrm{m}^{2}$ ) (VELARDE; DALMAU, 2012).
Para calcular o escore global de bem-estar de uma determinada granja devem ser seguidos três passos: i) coletar os dados das diferentes medidas de bem-estar relacionadas com os 12 critérios, que são expressas em pontuações (como exemplo, as lesões de vulva podem ser classificadas da seguinte forma: escore $0=$ animal sem lesão, escore $1=$ animal com lesão pequena, e escore $2=$ animal com lesão severa); ii) coletar as pontuações de cada um dos 12 critérios dos quatro princípios de bemestar; iii) combinar as pontuações dos princípios em uma pontuação global (BOTREAU et al., 2007b; WELFARE QUALITY ${ }^{\circledR}$ 2009). Na Figura 1 estão demonstradas as sequências dos passos para se obter a pontuação global do projeto Welfare Quality ${ }^{\circledR}$. 
Figura 1. Os três passos para pontuação global do projeto Welfare Quality ${ }^{\circledR}$.

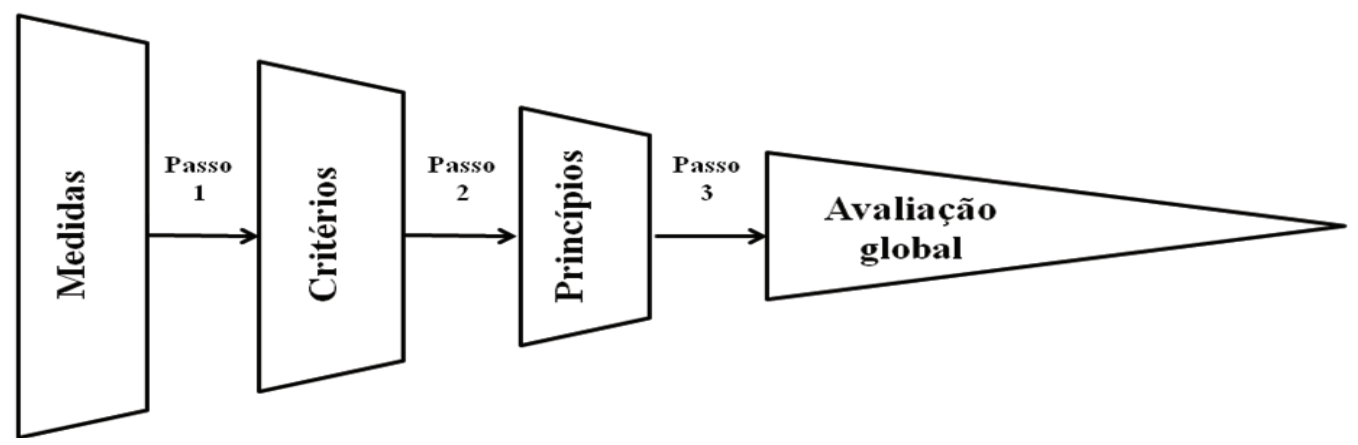

Fonte: Adaptado Welfare Quality ${ }^{\circledR}$ (2009).

As pontuações das medidas de um mesmo critério, quando combinadas, formam a pontuação final deste critério, obedecendo a uma escala de zero a 100. No nível zero entende-se que os problemas de bem-estar são máximos. $\mathrm{Na}$ escala 100 há a plenitude, a melhor condição de bem-estar. Da mesma forma, as pontuações obtidas nos distintos critérios, combinados, formam a pontuação dos quatro princípios (novamente é empregada a escala de zero a 100) (TEMPLE et al., 2009). As pontuações das medidas e dos critérios são então relacionadas de modo ponderal de acordo com sua importância, previamente definida através de painéis científicos. Como exemplo, a ausência de uma enfermidade é considerada uma situação mais importante que ausência de injúrias, que por sua vez é mais importante que a ausência de dor induzida por procedimentos de manejo (BOTREAU et al., 2007b).
Portanto, o escore global se obtém combinando de forma ponderada a pontuação dos quatro princípios (zero a 100), o qual classifica as unidades em uma das quatro categorias de bem-estar de acordo com um mínimo de pontuação obtido nos protocolos do projeto Welfare Quality ${ }^{\circledR}$. No entanto, os resultados altos em um dos princípios de bemestar não compensam os baixos em outros. Assim, as pontuações finais não podem se basear em pontuações médias (Figura 2). Para a caracterização de uma granja como excelente, a mesma deveria atingir mais de 55 pontos nos quatro princípios e mais de 80 em um deles. Uma granja classificada como boa deveria somar mais de 20 pontos nos quatro princípios e mais de $55 \mathrm{em}$ dois. Na qualidade de aceitável seriam necessários mais de 10 pontos nos quatro princípios e mais de 20 em três. As granjas que não atingem estas pontuações mínimas são consideradas não classificadas (WELFARE QUALITY $\left.^{\circledR}, 2009\right)$. 
Figura 2. Exemplos de granjas nas quatro categorias de bem-estar do projeto Welfare Quality ${ }^{\circledR}$.

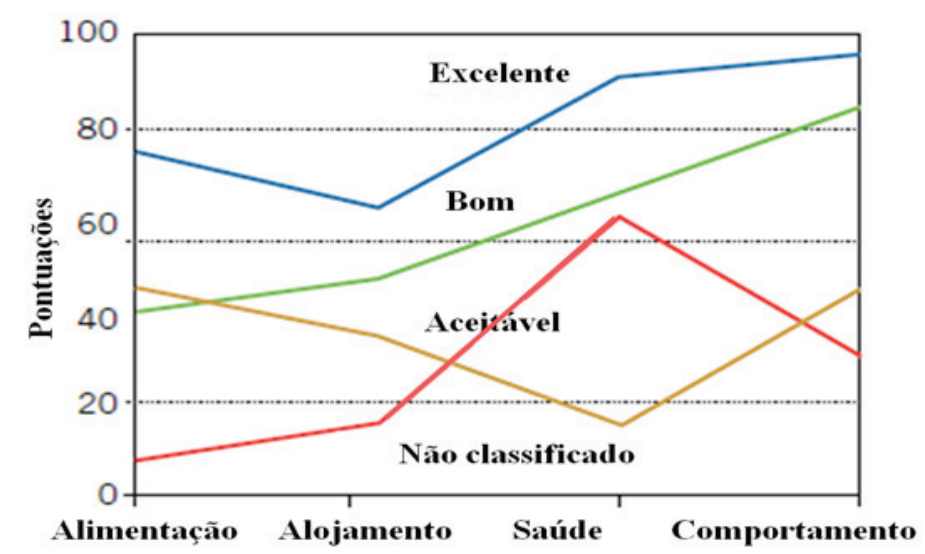

Fonte: Adaptado Welfare Quality ${ }^{\circledR}$ (2009).

O sistema de avaliação de bem-estar animal do projeto Welfare Quality ${ }^{\circledR}$ pode ser aplicado com vários propósitos. Após a avaliação das medidas nas granjas e nos abatedouros, as informações geradas podem ser utilizadas como estratégias de melhoria, corrigindo falhas e incrementando os processos, ou podem servir de informações para os consumidores (BLOKHUIS et al., 2003) (Figura 3).

Figura 3. Diagrama das possíveis aplicações de estratégias com o uso dos protocolos Welfare Quality ${ }^{\circledR}$.

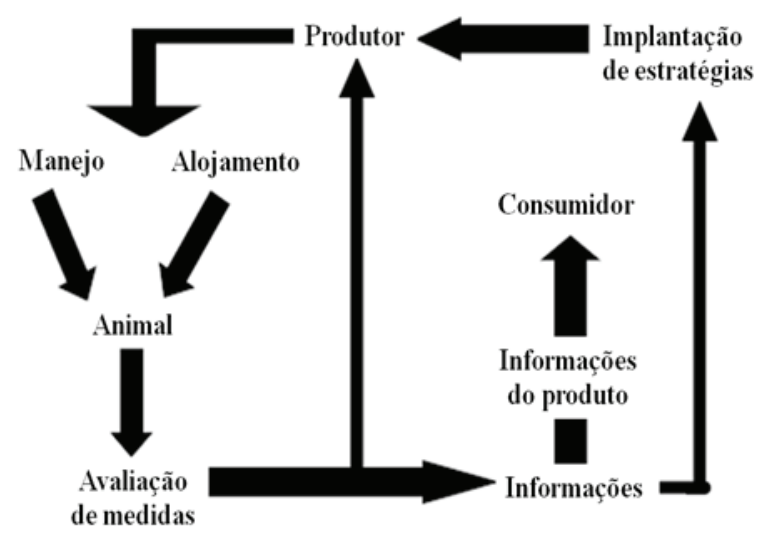

Fonte: Adaptado Blokhuis et al. (2003).

\section{Resultados das Avaliações do Projeto Welfare Quality $^{\circledR}$}

Concebido o projeto Welfare Quality ${ }^{\circledR}$, os protocolos foram aplicados primeiramente na Europa com foco na validação da metodologia em granjas comercias, envolvendo matrizes e leitões lactentes, em unidades de crescimento e em abatedouros.
A aplicação do protocolo Welfare Quality ${ }^{\circledR}$ para porcas e leitões demandou entre 4 a 5 horas por avaliação, sendo observadas que algumas medidas não se mostraram práticas em granjas que adotavam o sistema outdoor de criação. Neste sistema, a aproximação às fêmeas é dificultada não permitindo condições suficientes para realizar os exames individuais, como também avaliar as medidas de 
comportamento sem alteração do comportamento do grupo. Além disso, a tradicional coleta de dados com caneta e papel pode ser dificultada pelo clima. Os aspectos positivos encontrados foram que as medidas não se mostraram invasivas e não houve necessidade de movimentação dos animais das baias (SCOTT et al., 2009).

A aplicação completa do protocolo na fase de crescimento em 30 granjas intensivas espanholas levou em média 6 horas e 20 minutos, e muitos parâmetros usados no protocolo apresentaram alta variabilidade, permitindo desta forma diferenciar as granjas. Contudo, as causas desta variabilidade mostraram-se difíceis de serem interpretadas, especialmente as relacionadas com as medidas de comportamento. Esta metodologia pode ser aplicada para identificar granjas com problemas específicos de bem-estar, especialmente para as medidas baseadas no animal, que apresentaram baixa prevalência. Em síntese, este sistema de avaliação não permite definir o que poderia ser considerado como ruim ou bom bem-estar, mas é uma forma prática para detectar granjas com mais deficiências neste tema (TEMPLE et al., 2011).

A aplicação da metodologia do protocolo Welfare Quality ${ }^{\circledR}$ na fase de crescimento, em 91 granjas comerciais, em cinco sistemas de produção da França e Espanha, demonstrou que em geral as medidas baseadas no animal, considerando o princípio da boa saúde, diferiram pouco entre os sistemas. Os sistemas extensivos mostraram menor prevalência de feridas severas no corpo e de caudofagia, comparado com o sistema convencional confinado, sendo atribuídos estes achados, em maior ou menor frequência, aos modelos de alimentação e tipo de pisos empregados, indicando que o simples registro dos fatores baseados no ambiente pode ser útil para detectar granjas com maior probabilidade de mostrar estes problemas (TEMPLE et al., 2012a). Importantes diferenças entre os sistemas de produção puderam ser estabelecidas analisando as medidas baseadas no animal, considerando os princípios da boa alimentação e do bom alojamento, todavia, alguns registros de medidas baseadas no ambiente, como tipo de piso, sistema de alimentação, densidade e temperatura ambiente, também podem ser úteis para identificar granjas com maiores risco de deficiências relacionadas com o princípio do bom alojamento (TEMPLE et al., 2012b).

Quanto a viabilidade da aplicação do protocolo Welfare Quality ${ }^{\circledR}$ em abatedouros, Dalmau et al. (2009) demonstraram que é possível qualificar o estado de bem-estar nestas unidades estabelecendo um escore entre os piores e os melhores, além de também identificar problemas nas áreas específicas destas plantas. Utilizando dez unidades de abate, a média de tempo para realizar a observação completa, conforme prescreve o protocolo, foi de cinco horas e meia. Os dois principais fatores que podem afetar o tempo de realização do protocolo em abatedouros são a frequência da chegada das cargas e o tempo entre o descarregamento e o início do abate (VELARDE; DALMAU, 2012).

Nestas unidades, como também nos demais sistemas, um componente crítico na avaliação do bem-estar animal é a qualidade dos avaliadores. Os mesmos devem receber treinamentos para uniformizarem as avaliações que demandam atribuições mais subjetivas, como os escores; devem possuir um bom entendimento da biologia, da fisiologia, da saúde, do comportamento animal e dos mecanismos que os suínos recorrem para se adaptar a determinados ambientes; e finalmente devem ser capazes de reconhecer sintomas de certas lesões e enfermidades (VELARDE; DALMAU, 2012).

São inegáveis os avanços obtidos na ciência do bem-estar animal com o projeto Welfare Quality ${ }^{\circledR}$. No entanto, as aplicações dos protocolos em condições industriais geraram algumas críticas, sendo que a medida mais questionada dos protocolos é o Qualitative Behaviour Assessment (QBA), um parâmetro subjetivo utilizado para avaliar o estado emocional positivo. Esta metodologia 
utiliza observações que descrevem a qualidade da linguagem corporal do animal, sendo aplicados termos como ansioso, calmo e agressivo. No entanto, a comunidade científica é dividida sobre a validade dos resultados da QBA, pois encontra dificuldade em aceitar que a linguagem corporal forneça uma transparente "janela" com a mente animal, não sendo considerada uma metodologia segura para avaliação do bem estar mental (FAWC, 2009). A aplicação da metodologia do QBA somente é válida para ordenar granjas comerciais em relação ao estado emocional (positivo), quando analisados os dados de uma única visita, pois na experiência de Temple et al. (2013), o escore do QBA não se apresentou estável/confiável entre duas mensurações desenvolvidas nas mesmas granjas.

Finalmente, o projeto Welfare-Quality ${ }^{\circledR}$ gerou as bases para o desenvolvimento de um sistema de certificação, mediante a elaboração de indicadores científicos baseados nos animais, que poderá fundamentar a geração de informações transparentes e fiáveis aos consumidores sobre a classificação dos produtos (UNIÓN EUROPEA, 2011). O estabelecimento de um selo para bem-estar animal completaria este conjunto, apontando e dirigindo a cadeia para produção de produtos elaborados oriundos de condutas que dispensam um alto nível de bem-estar aos animais (VELARDE; DALMAU, 2012).

\section{Conclusões}

O bem-estar é um conceito multidimensional, e por esta razão uma única medida isolada não pode ser usada como um indicador de bem-estar.

A orientação das medidas para o próprio animal, mais do que para o seu entorno, objetiva melhorar as avaliações práticas do bem-estar.

A qualidade do trabalho do avaliador é um ponto crítico para o sucesso de qualquer metodologia de mensuração do bem-estar.

\section{Referências}

BARNETT, J. L.; HEMSWORTH, P. H. The validity of physiological and behavioural measures of animal welfare. Applied Animal Behaviour Science, Amsterdam, v. 25, p. 177-187, 1990.

BAXTER, E. M.; LAWRENCE, A. B.; EDWARDS, S. A. Alternative farrowing systems: design criteria for farrowing systems based on the biological needs of sows and piglets. Animal, Cambridge, v. 5, n. 4, p. 580-600, 2011.

BERGERON, R.; MEUNIER-SALAUN, C.; ROBERT, $\mathrm{S}$. The welfare of pregnant and lactating sows. In: FAUCITANO, L.; SCHAEFER, A. Welfare of pigs from birth to slaughter. Versailles: Wageningen Academic Publishers, 2008. p. 65-95.

BLOKHUIS, H. J. International cooperation in animal welfare: the Welfare Quality ${ }^{\circledR}$ project. Acta Veterinaria Scandinavica, Copenhagen, v. 50, p. 1-5, 2008. Supplement 1.

BLOKHUIS, H. J. Welfare Quality ${ }^{\circledR}$ : context, progress and aims. In: ASSURING ANIMAL WELFARE: FROM SOCIETAL CONCERNS TO IMPLEMENTATION, SECOND WELFARE QUALITY STAKEHOLDER CONFERENCE, 2007, Berlin. Proceedings... Berlin: [s.n], 2007. p. 9-12.

BLOKHUIS, H. J.; JONES, R. B.; GEERS, R.; MIELE, M.; VEISSIER, I. Measuring and monitoring animal welfare: transparency in the food product quality chain. Animal Welfare, Wheathampstead, v. 12, n. 4, p. 445-455, 2003.

BOTREAU, R.; BONDE, M.; BUTTERWORTH, A.; PERNY, P.; BRACKE, M. B. M.; CAPDEVILLE, J.; VEISSIER, I. Aggregation of measures to produce an overall assessment of animal welfare. Part 1 - a review of existing methods. Animal, Cambridge, v. 1, n. 8, p. 1179-1187, 2007a.

BOTREAU, R.; BRACKE, M. B. M.; PERNY, P.; BUTTERWORTH, A.; CAPDEVILLE, J.; VAN REENEN, C. G.; VEISSIER, I. Aggregation of measures to produce an overall assessment of animal welfare. Part 2: analysis of constraints. Animal, Cambridge, v. 1, n. 8, p. 1188-1197, 2007b.

BRACKE, M.; VISSER-RIEDSTRA, K.; SCHEPERS, F.; URSINUS, N.; BLOKHUIS, H; GERRITZEN M.; GAST, E. Scientific and technological state-of-the-art. In: BOKMA-BAKKER, M.; MUNNICHS, G. Animalbased welfare monitoring final report. The Netherlands: Rathenau Institute, 2009. p. 1-38. 
BROOM, D. M. Animal welfare: concepts, study methods and indicators. Revista Colombiana de Ciencias Pecuarias, Medellin, v. 24, n. 3, p. 306-321, 2011.

Coping, stress, and welfare. In: BROOM, D. M. Coping with challenge: welfare in animals including humans. Berlim: Dahlem University Press, 2001b. p. 1-9.

Indicators of poor welfare. British Veterinary Journal, London, v. 142, p. 524-526, 1986.

The use of the concept animal welfare in european conventions, regulations and directives. Food Chain, Uppsala, p. 148-151, 2001a.

Welfare concepts. In: FAUCITANO, L.; SCHAEFER, A. Welfare of pigs from birth to slaughter. Versailles: Wageningen Academic Publishers, 2008. p. 15-32.

BROOM, D. M.; JOHNSON, K. G. Stress and animal welfare. Dordrecht: Kluver Academic Publishers, 1993. $138 \mathrm{p}$.

BROOM, D. M.; MOLENTO, C. F. M. Bem-estar animal: conceito e questões relacionadas - revisão. Archives of Veterinary Science, Curitiba, v. 9, n. 2, p. 1-11, 2004.

DALMAU, A.; TEMPLE, D.; RODRÍGUEZ, P.; LLONCH, P.; VELARDE, A. Application of the Welfare Quality ${ }^{\circledR}$ protocol at pig slaughterhouses. Animal Welfare, Wheathampstead, v. 18, n. 4, p. 497-505, 2009.

DAWKINS, M. S. Behavioural deprivation: a central problem in animal welfare. Applied Animal Behaviour Science, Amsterdam, v. 20, n. 3-4, p. 209-225, 1988.

DUNCAN, I. J. H. Animal welfare defined in terms of feelings. Acta Agriculturae Scandinavica, Copenhagen, p. 29-35, 1996. Supplement 27.

Science-based assessment of animal welfare: farm animals. Revue Scientifique et Technique International Office of Epizootics, Paris, v. 24, n. 2, p. 483-492, 2005.

DUNCAN, I. J. H.; FRASER, D. Understanding animal welfare. In: APPLEBY, M. C.; HUGHES, B. O. Animal welfare. London: Ed. Cab International, 1997. p. 19-31.

EFSA. Scientific opinion statement on the use of animalbased measures to assess the welfare of animals. The EFSA Journal, Parma, v. 10, n. 6, p. 29, 2012b.

Scientific opinion on the use of animal-based measures to assess welfare in pigs. The EFSA Journal, Parma, v. 10, n. 1, p. 85, 2012a.

FARM ANIMAL WELFARE COUNCIL - FAWC. Farm animal welfare in great britain: past, present and future. London, 2009. p. 1-59.
FRASER, D. Science, Values and animal welfare: exploring the 'inextricable connection'. Animal Welfare, Wheathampstead, v. 4, n. 2, p. 103-117, 1995.

GRANDIN, T. Auditing animal welfare at slaughter plants. Meat Science, Barking, v. 86, n. 1, p. 56-65, 2010.

GUYTON, A. C.; HALL, J. E. Tratado de fisiologia médica. 9. ed. México: Mac Graw-Hill Interamericana, 1997. $1262 \mathrm{p}$.

HUGHES, B. O. The historical and ethical background of animal welfare. How well do our animals fare? In: ANNUAL CONFERENCE OF THE READING UNIVERSITY AGRICULTURAL CLUB, 15., E. J.Uglow, 1982. Proceedings ... E. J.Uglow: [s.n], 1982. p. 1-9.

HURNIK, J. F. Behaviour. In: PHILLIPS, C.; PIGGINS, D. Farm animals and the environment. Wallingford: Ed. C.A.B. International, 1992. p. 235-244.

Welfare of farm animals. Applied Animal Behaviour Science, Amsterdam, v. 20, n. 1-2, p. 105-117, 1988.

KEELING, L.; VEISSIER, I. Developing a monitoring system to assess welfare quality in cattle, pigs and chickens. In: SCIENCE AND SOCIETY IMPROVING ANIMAL WELFARE. WELFARE QUALITY CONFERENCE, 2005, Brussels. Proceedings... Brussels: [s.n.], 2005. p. 46-50.

MANTECA, X. Bienestar animal en explotaciones de porcino. In: CONGRESSO BRASILEIRO DE VETERINÁRIOS ESPECIALISTA EM SUÍNOSABRAVES, 15., 2011, Fortaleza. Anais... Fortaleza: ABRAVES, 2011. p. 13-17.

MANTECA, X.; GASA, J. Bienestar en el ganado porcino. Barcelona: Boehringer Ingelheim España, S.A., 2008. $118 \mathrm{p}$.

MANTECA, X.; VELARDE, A.; JONES, B. Animal welfare components. In: SMULDERS, F.; ALGERS, B. Welfare of production animals: assessment and management of risks. Wageningen, 2009. p. 61-77.

McGLONE, J. J.; von BORELL, E. H.; DEEN, J.; JOHNSON, A. K.; LEVIS, D. G.; MEUNIER-SALAÜN, M.; MORROW, J.; REEVES, D.; SALAK-JOHNSON, J. L.; SUNDBERG, P. L. Compilation of the scientific literature comparing housing systems for gestating sows and gilts using measures of physiology, behavior, performance, and health. The Professional Animal Scientist, Champaign, v. 20, n. 2, p. 105-117, 2004.

MIELE, M.; VEISSIER, I.; EVANS, A.; BOTREAU, R. Animal welfare: establishing a dialogue between science and society. Animal Welfare, Wheathampstead, v. 20, p. 103-117, 2011. 
OIE. World Organisation for Animal Health. Terrestrial animal health code. 18. ed. Paris: World Organization for Animal Health, 2009. v. 1, 444 p.

POOLE, T. Natural behaviour is simply a question of survival. Animal Welfare, Wheathampstead, v. 5, p. 218, 1996.

RUSHEN, J. Problems associated with the interpretation of physiological data in the assessment of animal welfare. Applied Animal Behaviour Science, Amsterdam, v. 28, n. 4, p. 381-386, 1991.

SCOTT, K.; BINNENDIJK, G. P.; EDWARDS, S. A.; GUY, J. H.; KIEZEBRINK, M. C.; VERMEER, H. M. Preliminary evaluation of a prototype welfare monitoring system for sows and piglets (Welfare Quality ${ }^{\circledR}$ project). Animal Welfare, Wheathampstead, v. 18, n. 4, p. 441-449, 2009.

TEMPLE, D.; COURBOULAY, V.; VELARDE, A.; DALMAU, A.; MANTECA, X. The welfare of growing pigs in five different production systems in France and Spain: assessment of health. Animal Welfare, Wheathampstead, v. 21, n. 2, p. 257-271, 2012a.

TEMPLE, D.; COURBOULAY, V.; VELARDE, A.; MANTECA, X.; DALMAU, A. The welfare of growing pigs in five different production systems: assessment of feeding and housing. Animal, Cambridge, v. 6, n. 4, p. 657-667, 2012b.

TEMPLE, D.; DALMAU, A.; RUÍZ de la TORRE, J. L.; MANTECA, X.; VELARDE, A. Application of the
Welfare Quality protocol to assess growing pigs kept under intensive conditions in Spain. Journal of Veterinary Behavior, Canadá, v. 6, n. 2, p. 138-149, 2011.

TEMPLE, D.; MANTECA, X.; DALMAU, A.; VELARDE, A. Assessment of test-retest reliability of animal-based measures on growing pig farms. Livestock Science, London, v. 151, n. 1, p. 35-45, 2013.

TEMPLE, D.; VELARDE, A.; MANTECA, X.; DALMAU, A. Evaluación de bienestar mediante el protocolo Welfare Quality ${ }^{\circledR}$ en el cerdo ibérico en extensivo: resultados preliminares. In: Especial "Solo Cerdo Ibérico”, Tomo I, Badajoz, p. 384-393, 2009.

UNIÓN EUROPEA. Dictamen del Comité Económico y Social Europeo sobre el Informe de la Comisión al Parlamento Europeo, al Consejo, al Comité Económico y Social Europeo y al Comité de las Regiones: Opciones de etiquetado del bienestar animal y establecimiento de una Red Europea de Centros de Referencia para la Protección y el Bienestar de los Animales. COM (2009) 584 final (2011/C 21/08). Diario Oficial [de la] Unión Europea, Bruselas, 21 enero 2011. Sección 21, p. 44-48.

VELARDE, A.; DALMAU, A. Animal welfare assessment at slaughter in Europe: moving from inputs to outputs. Meat Science, Barking, v. 92, n. 3, p. 244-251, 2012.

WELFARE QUALITY ${ }^{\circledR}$ assessment protocol for pigs (sows and piglets, growing and finishing pigs). Welfare Quality ${ }^{\circledR}$ Consortium, Lelystad, version 1, 2009. 122 p. 
